\title{
Inervación del Músculo Flexor Superficial de los Dedos
}

\author{
Innervation of the Superficial Flexor Digitorum Muscle
}

Riveros, A. ${ }^{1,2}$; Sousa-Rodrigues, C.F. ${ }^{3}$ \& Olave, E. ${ }^{4}$

\begin{abstract}
RIVEROS, A.; SOUSA-RODRIGUES. C. F. \& OLAVE, E. Inervación del músculo flexor superficial de los dedos. Int. J. Morphol., 36(2):768-777, 2018.

RESUMEN: La inervación del músculo flexor superficial de los dedos (FSD) es controversial. Diversos autores describen que los ramos de este músculo surgen independiente o desde un tronco común que proviene desde el nervio mediano, dentro de la región cubital. Otras descripciones señalan la presencia de ramos adicionales en los tercios medio y distal del antebrazo. Estas diferencias también se reflejan al describir los puntos motores. El objetivo fue determinar el número, ubicación y patrones de distribución de los ramos para el FSD. Se utilizaron 30 antebrazos de cadáveres adultos brasileños formolizados. En relación al número de ramos, el $26 \%$ presentó un solo ramo (R1). El 57 \% presentó dos ramos (R1 y R2) y el 17 \% presentó 3 ramos (R1, R2 y R3). Respecto al formato de origen, el $87 \%$ de los R1 surgieron independiente, mientras que el $13 \%$ surgió desde un tronco común. En el caso de los R2 y R3, el 100 $\%$ surgió independiente. En relación a los puntos de origen, el $90 \%$ de los R1 se originó en el tercio proximal del antebrazo, en tanto, el $60 \%$ de los R2 surgieron en el tercio medio, y de los 5 R3, el $60 \%$ se originó en el tercio medio y los dos restantes surgieron en el tercio distal. Esta misma variabilidad se presentó en la distribución de los puntos motores. Respecto a los patrones de distribución de estos ramos motores se identificaron de dos tipos. El patrón I se presentó en el 85 \% y se caracterizó por que todos sus ramos surgieron en un punto distal al origen del nervio interóseo anterior (NIA), en cambio, en el patrón tipo II (15\% de la muestra), el primer ramo se originó proximal al origen del NIA.
\end{abstract}

PALABRAS CLAVE: Anatomía; Nervio mediano; Puntos motores; Músculo flexor superficial de los dedos.

\section{INTRODUCCIÓN}

El músculo flexor superficial de los dedos es un músculo ubicado en el compartimiento anterior del antebrazo y es inervado, a nivel de la región cubital, por un ramo que se dirige desde el nervio mediano a los músculos de la capa superficial de este compartimiento muscular. Dicho ramo se divide en varios ramos menores que se dirigen a los músculos pronador redondo, flexor radial del carpo, palmar largo y flexor superficial de los dedos. (Cruveilhier, 1851; Testut \& Latarjet, 1972; Rouvière \& Delmas, 2005). Otros autores como Sappey (1889) y Poirier \& Charpy (1904), señalaron que el número de ramos que se originan de la mitad proximal de la porción antebraquial del nervio mediano, es desconocido, a lo que se suma, que la forma en la que estos ramos surgen, puede ser en ocasiones independientes o a través de uno o dos troncos comunes.

Al respecto, Linell (1921), entregó datos precisos respecto a la distribución de los ramos musculares del nervio mediano y su variabilidad de origen y penetración al músculo, concluyendo que los músculos flexor radial del carpo, palmar largo y flexor superficial de los dedos estaban inervados por un tronco común que se originó en promedio a $2 \mathrm{~cm}$ distal a la línea biepicondilar (LBE). Durante este trayecto, este tronco nervioso se dividió en un grupo de ramos secundarios, de los cuales, las fibras distales se dirigieron al músculo flexor superficial de los dedos. Así mismo para Canovas et al. (1998), el ramo destinado al músculo flexor superficial de los dedos era el ramo que presentaba más variabilidad en su origen, ya que en el $20 \%$ de los casos se originó de forma aislada desde el nervio mediano, en $20 \%$ desde el nervio interóseo anterior y en el $60 \%$, compartió origen con los ramos destinados a los otros músculos superficiales de este compartimiento. Independiente de la forma en la que se originó, su punto promedio de origen estuvo en los 3,5 cm distal a la LBE. Misma variabilidad expuso Sunderland \& Ray (1946), destacando que en el

\footnotetext{
${ }^{1}$ Departamento de Ciencias Morfológicas, Facultad de Medicina y Ciencia, Universidad San Sebastián, Lientur 1457, Concepción, 4080871, Chile.

${ }^{2}$ Programa de Doctorado en Ciencias Morfológicas, Universidad de La Frontera, Temuco, Chile

${ }^{3}$ Universidad Estadual de Ciencias da Saude de Alagoas, Maceió, Brasil.

${ }^{4}$ Facultad de Medicina, Universidad de La Frontera, Temuco, Chile.
} 
$65 \%$ de los casos el músculo flexor superficial recibió entre los 3 y 7 ramos. Así mismo, en el $70 \%$ de los casos, estos ramos se originaron del nervio mediano, dejando al $30 \%$ restante como aquellos en donde sus ramos surgieron desde el nervio interóseo anterior. Así mismo, en el $75 \%$ de los casos, este ramo surgió desde un tronco común con ramos para otros músculos, siendo el caso más frecuente, el que describió un origen común con el músculo flexor radial del carpo (35\% de los casos). En este sentido, Fuss \& Wurzl (1990), expuso que el $56 \%$ de su muestra, el nervio mediano se originó un tronco común para inervar los músculos palmar largo, flexor radial del carpo y flexor superficial de los dedos. A lo anterior se agrega lo expuesto por Liu et al. (1997), quienes señalaron que el conocimiento actual de la inervación del antebrazo es insuficiente para la práctica quirúrgica, este argumento se fundamenta en la gran variación respecto a la localización tanto de los puntos de origen como los puntos motores. Al respecto, estos autores señalaron que músculo flexor superficial de los dedos presentó una media de 4 ramos, los cuales se originaron en un $90 \%$ desde el nervio mediano, y en el 10 $\%$ surgieron en forma simultánea desde el nervio mediano y del nervio interóseo anterior.

Para Cruveilhier, Poirier \& Charpy, Rauber (1898) y Rouvière \& Delmas, independiente de los ramos que inervan la región proximal del músculo flexor superficial de los dedos, el nervio mediano proporciona dos o tres ramos adicionales, que se desprenden gradualmente del nervio mediano en su trayecto dentro del antebrazo. Esta descripción fue también expuesta por Canovas et al., en donde se identificaron ramos adicionales para el dicho músculo, los cuales surgieron del nervio mediano en el tercio distal del antebrazo. Así mismo Linell, identificó que en $46 \%$ de los casos, este músculo recibió ramos en la zona distal del antebrazo, agregando que estos ramos adicionales se vincularon con el vientre muscular que tiene este músculo tanto para el dedo índice como opcionalmente para el dedo medio. Para Williams et al. (1998), dicho ramo adicional surge del nervio mediano cerca de la mitad del antebrazo, pudiendo hacerlo también desde el nervio interóseo anterior. Al respecto, Llusá et al. (2013), descarta que existan ramos que surjan del nervio mediano en la región cubital. Así mismo describe que el músculo flexor superficial de los dedos recibe ramos en un punto distal a las cabezas de origen del citado músculo.

En relación a los patrones de distribución de los ramos motores que el nervio mediano entrega en el antebrazo, Liu et al. y Sunderland \& Ray señalaron que el patrón de distribución más frecuente describe un primer ramo para el músculo pronador redondo, un segundo ramo para el músculo palmar largo, seguido por el ramo para el músculo flexor radial del carpo. A continuación, aparece un número indeterminado de ramos para el músculo flexor superficial de los dedos, para distalmente dejar los ramos para los músculos flexor largo del pulgar y flexor profundo de los dedos.

\section{MATERIAL Y MÉTODO}

En el presente estudio se utilizaron 30 miembros superiores (16 derechos y 14 izquierdos) de individuos adultos, brasileños, pertenecientes a la Facultad de Medicina de la Universidad Estadual de Ciencias da Saúde de Alagoas, Maceió, Brasil. Estos cadáveres se encontraban fijados bajo inmersión en una solución con formaldehído al $10 \%$. De la totalidad de miembros.

Esta investigación se formuló bajo una metodología de carácter cuantitativo, no experimental, transeccional y descriptiva, para lo cual se procedió a realizar una detallada disección tanto de la región cubital como del compartimento anterior del antebrazo, utilizando para ello material quirúrgico "ad-hoc". Se determinó el origen de cada ramo para los músculos del compartimiento anterior del antebrazo, con especial énfasis en el músculo flexor superficial de los dedos. En cada ramo se analizó si su origen era independiente o provenía de un tronco común, además se registró la distancia de los puntos de origen y motor respecto a la LBE y a la línea biestiloidea (LB) (Fig. 1).

La disección se inició trazando dos incisiones horizontales, una proximal a la altura de la LBE, y otra distal, a la altura de la línea LB. En seguida estas incisiones horizontales fueron conectadas por una incisión longitudinal en la superficie anterior del antebrazo. Se procedió a retirar la piel, se prescindió del tejido adiposo subcutáneo, la fascia superficial, los elementos vasculares de la región cubital y el lacertus fibroso. Posteriormente, se procedió a disecar los ramos que el nervio mediano entregó en el tercio distal del brazo y en el antebrazo. Adicionalmente se analizó el origen, trayecto y distribución de los ramos del nervio interóseo anterior. Las mediciones fueron realizadas con un caliper digital Mitutoyo® de $0,01 \mathrm{~mm}$ de precisión. Complementariamente las muestras disecadas fueron fotografiadas con una cámara digital Canon ${ }^{\circledR}$ Rebel XTI.

Para determinar los patrones de distribución de los ramos destinados al músculo flexor superficial de los dedos se consideró el origen de éstos respecto a una secuencia de localización de proximal a distal, comparando el origen de los diferentes ramos que el nervio mediano (o nervio interóseo anterior) generó para los músculos del compartimiento anterior del antebrazo. 


\section{RESULTADOS}

En relación al número de ramos que recibió este músculo, se registró que el $26 \%$ de los miembros examinados presentó solamente un ramo, el cual se denominó R1. El 57 $\%$ de los casos se presentaron dos ramos motores, uno de ellos fue R1 y al segundo se le denominó R2. El 17 \% restante de miembros disecados presentaron 3 ramos motores, a este último lo denominamos R3. Los hallazgos relacionados con aspectos biométricos como longitud y diámetro se encuentran resumido en la Tabla I.

Respecto a la forma en que surgió cada uno de estos ramos, en el caso de R1, se encontró que en el $87 \%$ de los casos se originó de forma independiente, (en uno de los casos, R1 se originó desde el nervio interóseo anterior); mientras que en el $13 \%$ restante, este ramo surgió desde un tronco común proveniente desde el nervio mediano, el cual fue compartido con: a) un ramo para el músculo flexor profundo de los dedos, b) un ramo para el músculo palmar largo, c) los ramos R1 para los músculos flexor radial del carpo y palmar largo y d) con los ramos R1 para los tres músculos que completan el plano superficial del compartimiento anterior del antebrazo. En el caso de los ramos R2 y R3, el 100 $\%$ de ellos surgió deforma independiente desde el nervio mediano.

El punto de origen de los ramos del músculo flexor superficial de los dedos se midió determinando la distancia entre la LBE y el punto en la cual los ramos R1, R2 y R3 surgieron desde el nervio de origen, ya sea nervio mediano o nervio interóseo anterior. $\mathrm{Al}$ respecto, el promedio alcanzado por la totalidad de ramos asociados a este músculo alcanzo los 89,89 mm $\pm 49,95 \mathrm{~mm}$. Los ramos R1 presentaron un promedio de distancia de origen de $57,33 \mathrm{~mm} \pm 24,27$ $\mathrm{mm}$. Cabe señalar que el $73 \%$ de estos ramos R1 surgieron después de que el nervio mediano diera origen al nervio interóseo anterior; a esto se sumó el hecho que, en uno de los casos, este ramo R1 surgió a 1,35 mm, proximal a la LBE. Así mismo, los ramos R1 que surgieron de forma independiente alcanzaron una distancia promedio de origen de 60,89 $\mathrm{mm} \pm 24,06 \mathrm{~mm}$, en cambio, los ramos R1 que se originaron desde un tronco común, presentaron una distancia promedio de $34,14 \mathrm{~mm} \pm 6,51 \mathrm{~mm}$. En el caso del ramo motor R2, el promedio de la distancia del punto de origen fue de $118,7 \mathrm{~mm} \pm 47,94 \mathrm{~mm}$, dejando a los casos que presentaron R3, como aquellos en la cual la distancia promedio alcanzó los 158,67 $\mathrm{mm} \pm 13,26 \mathrm{~mm}$. Los datos respecto al promedio de la distancia de origen de cada uno de los ramos, junto a las distancias mínima y máxima se encuentra resumidas en la Figura 1.

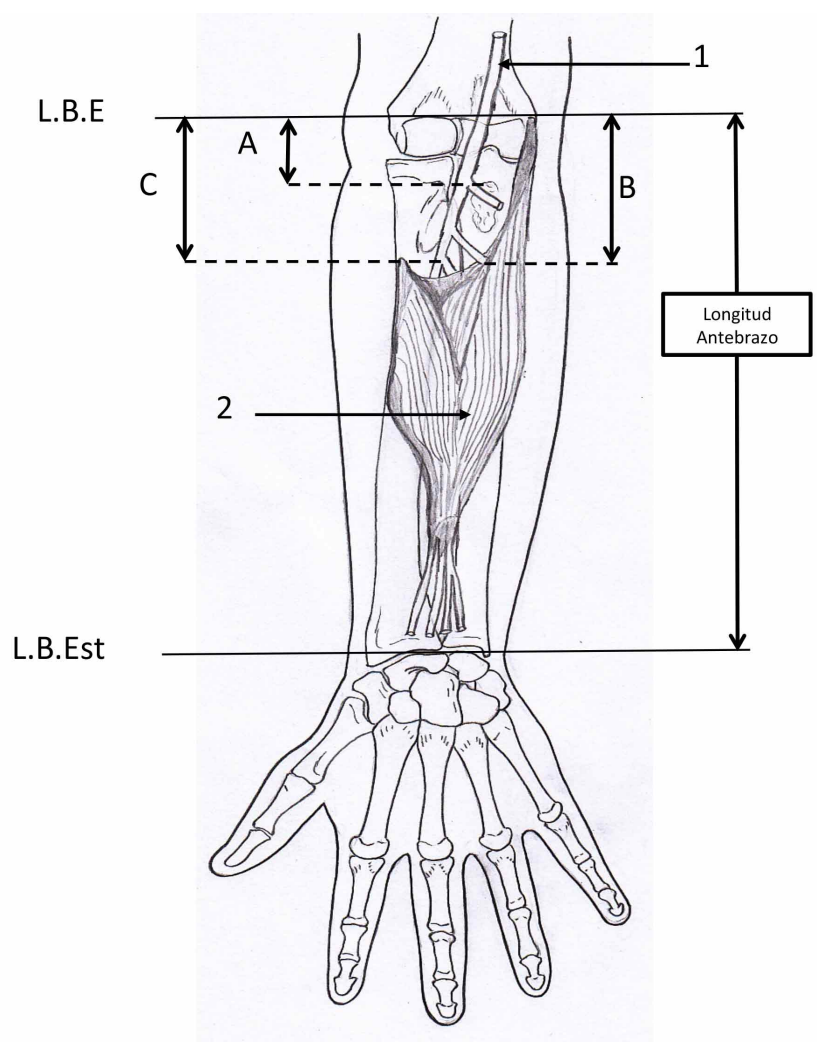

Fig. 1. Aspecto anterior del antebrazo. 1: Nervio mediano, 2: Músculo flexor superficial de los dedos, A: Distancia entre la LBE y el punto de origen del ramo motor, B: Distancia entre la LBE y el punto motor de ramo, C: Distancia entre la LBE y el punto de origen del nervio interóseo anterior, LBE: Línea biepicondilar, LB: Línea biestiloidea.

Tabla I: Biometría de los ramos para el músculo Flexor superficial de los dedos.

\begin{tabular}{lccccccccc}
\hline \multicolumn{1}{c}{ Longitud } & & \multicolumn{4}{c}{ Diámetro } \\
Ramo & $\mathrm{N}$ & Media (mm) & D.E. & Mín. (mm) & Máx. $(\mathrm{mm})$ & Media $(\mathrm{mm})$ & D.E. & Mín. (mm) & Máx. (mm) \\
\hline R1 & 30 & 25,47 & 11,63 & 8,76 & 50,75 & 1,44 & 0,64 & 0,59 & 3,91 \\
22 & 22 & 19,85 & 9,14 & 5,91 & 38,92 & 1,06 & 0,45 & 0,41 & 2,10 \\
23 & 5 & 24,23 & 6,34 & 14,1 & 29,17 & 1,13 & 0,69 & 0,59 & 1,95 \\
Total & 57 & 23,19 & 10,56 & 5,91 & 50,75 & 1,27 & 0,60 & 0,41 & 3,91 \\
\hline
\end{tabular}


El punto de origen de los ramos del músculo flexor superficial de los dedos se midió determinando la distancia entre la LBE y el punto en la cual los ramos R1, R2 y R3 surgieron desde el nervio de origen, ya sea nervio mediano o nervio interóseo anterior. $\mathrm{Al}$ respecto, el promedio alcanzado por la totalidad de ramos asociados a este músculo alcanzó los 89,89 $\mathrm{mm} \pm$ 49,95 $\mathrm{mm}$. Los ramos R1 presentaron un promedio de distancia de origen de $57,33 \mathrm{~mm} \pm 24,27 \mathrm{~mm}$. Cabe señalar que el $73 \%$ de estos ramos R1 surgieron después de que el nervio mediano diera origen al nervio interóseo anterior; a esto se sumó el hecho que, en uno de los casos, este ramo R1 surgió a 1,35 mm, proximal a la LBE. Así mismo, los ramos R1 que surgieron de forma independiente alcanzaron una distancia promedio de origen de $60,89 \mathrm{~mm} \pm$ $24,06 \mathrm{~mm}$, en cambio, los ramos R1 que se originaron desde un tronco común, presentaron una distancia promedio de $34,14 \mathrm{~mm} \pm 6,51 \mathrm{~mm}$. En el caso del ramo motor R2, el promedio de la distancia del punto de origen fue de $118,7 \mathrm{~mm} \pm 47,94 \mathrm{~mm}$, dejando a los casos que presentaron R3, como aquellos en la cual la distancia promedio alcanzó los 158,67 $\mathrm{mm} \pm 13,26 \mathrm{~mm}$. Los datos respecto al promedio de la distancia de origen de cada uno de los ramos, junto a las distancias mínima y máxima se encuentra resumidas en la Figura 2.

Además de establecer la distancia del punto de origen, se procedió a ubicar la proporción porcentual promedio entre el punto de origen y la longitud total del antebrazo. Los resultados de esta, establecieron que en el caso del ramo R1, este promedio se ubicó en los $22,74 \%$, dejando a los ramos R1 de origen aislado en el porcentaje $24,15 \%$ versus los ramos R1 que surgieron desde un tronco común que establecieron su proporción en el porcentaje 13,54\%. Respecto al ramo R2, la ubicación porcentual dentro de la longitud del antebrazo se ubicó en el 47,33\%, dejando al ramo R3 en un porcentaje de $65,72 \%$.

Otra de las formas utilizadas para establecer la localización de los puntos de origen consistió en determinar el tercio del antebrazo en la cual los diferentes ramos motores se originaron. Así fue como de la totalidad de ramos R1, el $90 \%$ se originó en el tercio proximal, siendo más preciso indicar que el $70 \%$ de éstos lo hizo en la mitad distal de este tercio. Bajo este mismo criterio es que se pudo observar que el $60 \%$ de los ramos R2 surgió en el tercio medio, del cual, el 54 $\%$ se originó en la mitad proximal de dicho tercio. Por último, de los 5 casos que presentaron ramo R3, 3 ubicaron su origen en la mitad distal del tercio medio, dejando a los dos restantes, como aquellos que surgieron de la mitad proximal del tercio distal.

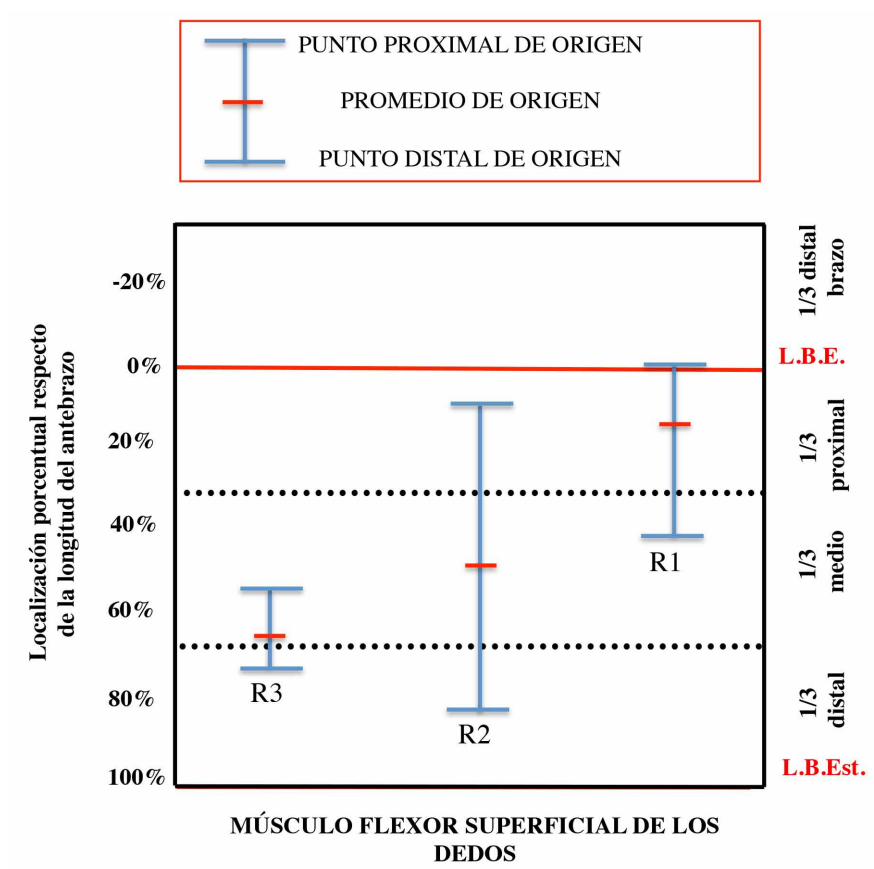

Fig. 2. Distancia del punto de origen de los ramos motores para el músculo flexor superficial de los dedos.

Respecto de los puntos motores de los ramos denominados R1, la distancia promedio de penetración del músculo flexor superficial de los dedos fue de 75,71 $\mathrm{mm} \pm 19,99 \mathrm{~mm}$. A lo anterior, se agregó la comparación entre esta distancia promedio de los ramos R1 que surgieron de forma independiente, versus los que surgieron desde un tronco común, encontrando que en el caso de los primeros, la distancia promedio fue de 78,95 $\mathrm{mm} \pm 19,11 \mathrm{~mm}$, en cambio, en el caso de los que se originaron desde un tronco común, la distancia promedio fue de 54,64 $\mathrm{mm} \pm 11,71 \mathrm{~mm}$. En relación a los casos R2 y $\mathrm{R} 3$ que en $100 \%$ de los casos se originaron de forma independiente, la distancia promedio entre el punto motor y la LBE, fue de $129,13 \mathrm{~mm} \pm 44,71 \mathrm{~mm}$, y los $168,72 \mathrm{~mm} \pm 10,29 \mathrm{~mm}$, respectivamente.

Para complementar estos aspectos vinculados con el punto motor es que observó el vientre muscular especifico que inervaron los ramos R2 y R3 correspondiente al músculo flexor superficial de los dedos, encontrando que en el $100 \%$ de los casos en la cual se presentaron estos ramos, su destino fueron los vientres musculares laterales, siendo preferente el vientre muscular que el músculo flexor superficial de los dedos entregó al dedo índice (Fig. 3).

Así mismo se procedió a establecer la proporción entre el punto motor de estos ramos y la longitud total del antebrazo, se determinó que el porcentaje que alcanzó el ramo R1 se ubicó en promedio en los 30,17\% de la longitud total del antebrazo. Así 


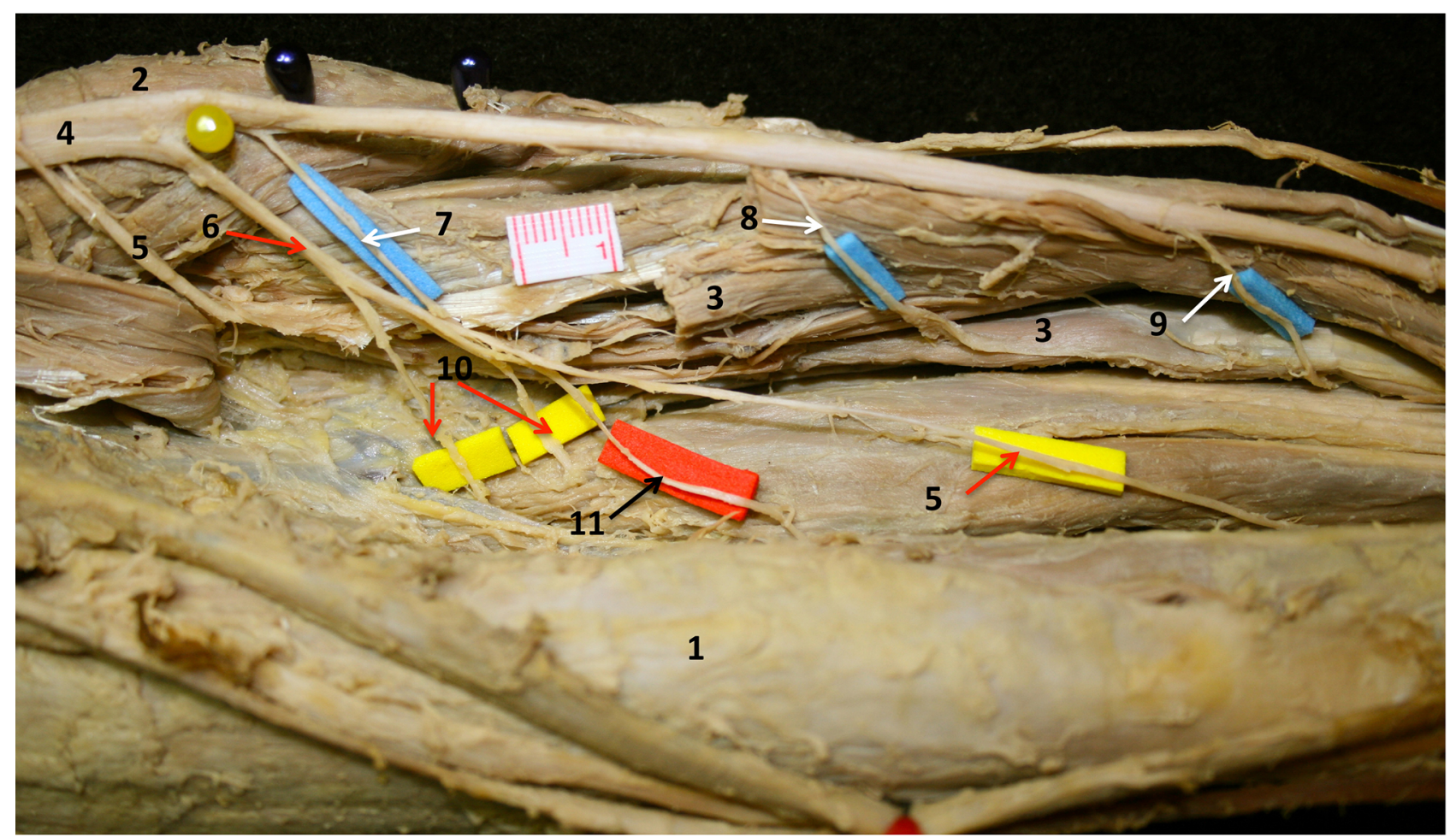

Fig. 3. Antebrazo derecho. 1: Músculo Braquiorradial, 2: Músculo Pronador Redondo, 3: Músculo Flexor Superficial de los dedos, 4: Nervio mediano, 5: Ramo motor para el músculo pronador redondo, 6: Nervio interóseo anterior, 7: Ramo motor R1 para el músculo flexor superficial de los dedos, 8: Ramo motor R2 para el músculo flexor superficial de los dedos, 9: Ramo motor R3 para el músculo flexor superficial de los dedos, 10: Ramos motores del músculo flexor profundo de los dedos, 11: Ramo motor del músculo flexor largo del pulgar.

mismo, es que en el caso de los ramos R2 y R3, la ubicación proporcional porcentual se ubicó en los $51,47 \%$ y en los $69,92 \%$, respectivamente.

En relación a la ubicación de este punto motor en alguno de los tercios del antebrazo, se encontró que el $66 \%$ de los ramos R1 penetró al músculo flexor superficial de los dedos en el tercio proximal del antebrazo, de los cuales, el $85 \%$ lo hizo en la mitad distal de dicho segmento. El ramo motor R2 fue el que presentó la mayor variabilidad. En el $63 \%$, el punto motor se ubicó en el tercio medio, de los cuales, el $57 \%$ inervó a este músculo en la mitad distal de dicho tercio. En el $60 \%$ de los 5 casos en donde se presentó el ramo motor R3, el punto motor se ubicó en la mitad distal del tercio medio, dejando a los dos restantes, como aquellos que ubicaron dicho punto motor en la mitad proximal del tercio distal del antebrazo.

Por último, y en base a la secuencia de los ramos que el nervio mediano generó para los músculos superficiales del compartimiento anterior del antebrazo, se estableció la presencia de 2 patrones de distribución los cuales están representados en las Figuras 4 y 5 .
Patrón Tipo I: Este patrón de distribución se presentó en el $85 \%$ de los casos y se caracterizó por presentar una secuencia de origen de los ramos que comenzó con el o los ramos destinados al músculo pronador redondo seguido de los ramos dirigidos a los músculos palmar largo y flexor radial de carpo. A lo anterior se suma al hecho de que en el $100 \%$ de los casos, los ramos del músculo flexor superficial de los dedos surgieron en un punto distal al origen del nervio interóseo anterior, el cual se originó en promedio a 53,92 mm, de la LBE. El ramo R1 para el músculo flexor superficial de los dedos presentó una distancia de origen promedio de $63,97 \mathrm{~mm}$, a lo que se agrega el hecho de que los ramos R2 y R3 para este músculo alcanzaron un promedio de origen de $130 \mathrm{~mm}$ y $161 \mathrm{~mm}$, distal a la LBE, respectivamente (Fig. 4).

Patrón Tipo II: Este formato de distribución se presentó en el $15 \%$ de la muestra, en donde la característica principal se asoció a que el primer ramo motor para el músculo flexor superficial de los dedos se originó en un punto proximal al origen del nervio interóseo anterior. En los 4 casos que conformaron este grupo el primer ramo que se originó desde el nervio mediano fue destinado al músculo pronador redon- 
do, el segundo músculo inervado fue el músculo palmar largo, cuyo ramo se originó desde un tronco común, el cual fue compartido en un $75 \%$ de los casos con el ramo para el músculo flexor radial del carpo. Un elemento distintivo de este patrón se asoció a que, en todos los casos, el ramo R1 para el flexor superficial de los dedos surgió proximal al origen del nervio interóseo anterior, siendo el promedio del primero de $39,88 \mathrm{~mm}$, distal a la LBE, versus $\operatorname{los} 40,63 \mathrm{~mm}$, del segundo. A esto se agrega que en el $75 \%$ de los casos el ramo R1 para el músculo flexor superficial de los dedos se originó desde un tronco común, el cual presentó un promedio de origen de $23,28 \mathrm{~mm}$, distal a la LBE. A lo anterior se agrega que en el $50 \%$ de los casos este músculo solo presentó ramo R1, dejando a los dos casos restantes como aquellos que presentaron ramo R2, e incluso una de ellos también presentó el ramo R3 (Fig. 5).

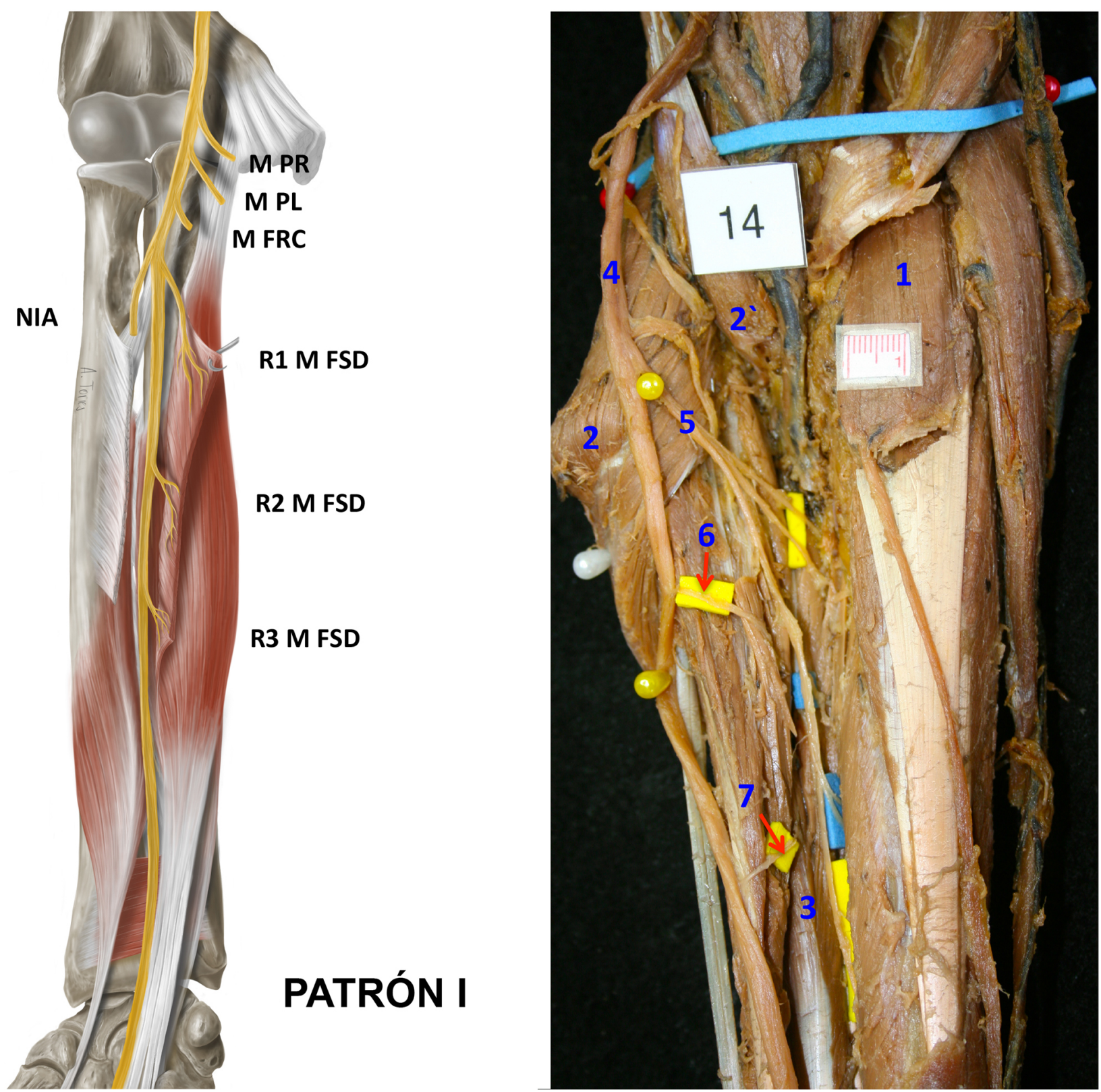

Fig. 4. Patrón I: M PR: Músculo pronador redondo, M PL: Músculo palmar largo, M FRC: Músculo flexor radial del carpo, M FSD: Músculo flexor superficial de los dedos, NIA: Nervio interóseo anterior, 1: Músculo Braquiorradial, 2: Músculo Pronador Redondo, cabeza humeral, 2: Músculo Pronador Redondo, cabeza ulnar, 3: Músculo Flexor Superficial de los dedos, 4: Nervio mediano, 5: Nervio interóseo anterior, 6: Ramo motor R1 para el músculo Flexor superficial de los dedos, 7: Ramo motor R2 para el músculo flexor superficial de los dedos. 


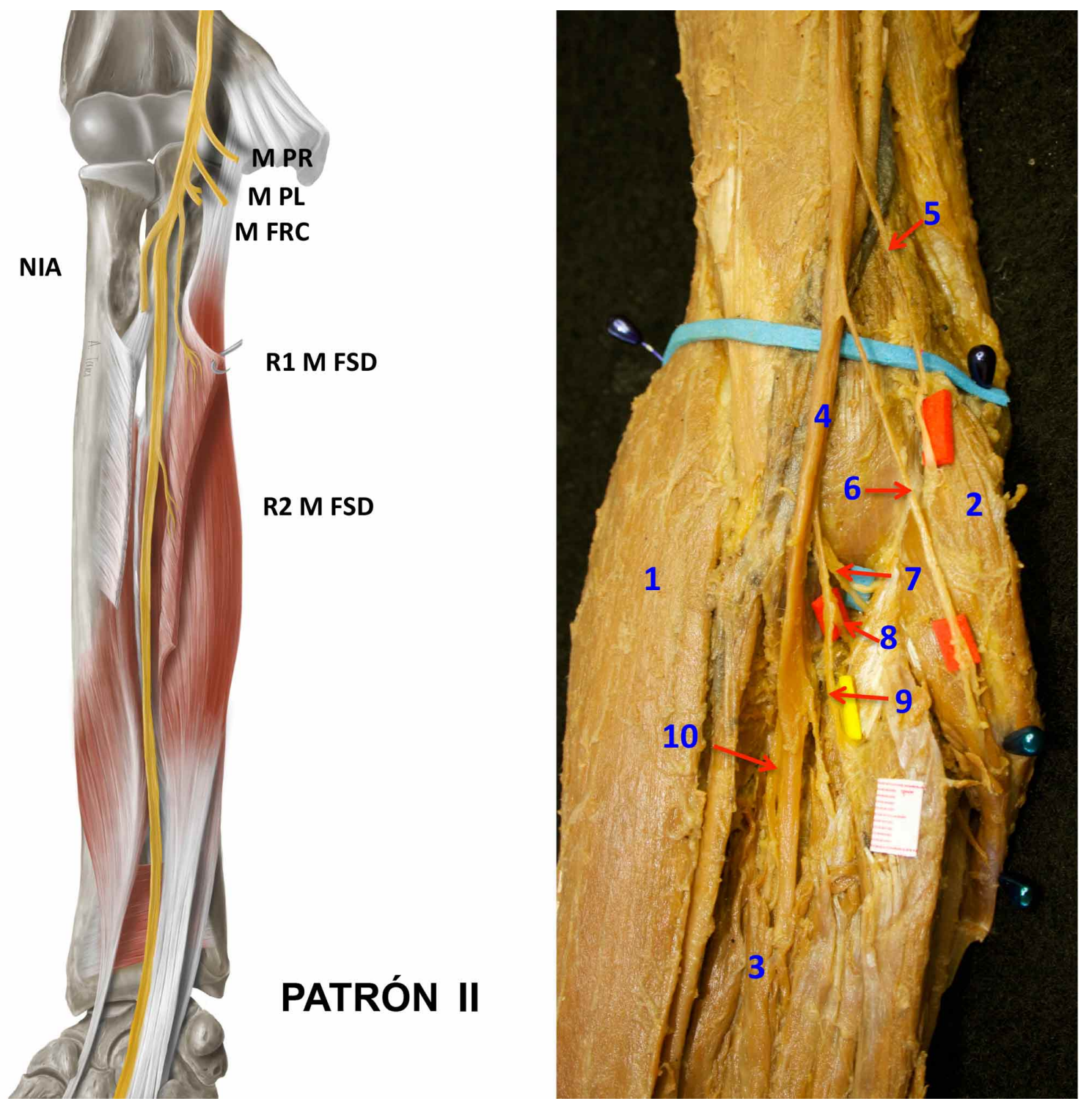

Fig. 5. Patrón II: M PR: Músculo pronador redondo, M PL: Músculo palmar largo, M FRC: Músculo flexor radial del carpo, M FSD: Músculo flexor superficial de los dedos, NIA: Nervio interóseo anterior, 1: Músculo braquioradial, 2 : Músculo pronador redondo, 3: Músculo flexor superficial de los dedos, 4: Nervio mediano, 5: Ramo motor R1 para el músculo pronador redondo, 6: Ramo motor R2 para el músculo Pronador redondo, 7: Ramo motor del músculo Palmar largo, 8: Ramo motor para el músculo flexor radial del carpo, 9: Ramo motor R1 para el músculo flexor superficial de los dedos, 10: Nervio interóseo anterior.

\section{DISCUSIÓN}

El músculo flexor superficial de los dedos es uno de los músculos del compartimento anterior del antebrazo con mayor variabilidad en su inervación, en este sentido, un 74 $\%$ de nuestra muestra presentó más de un ramo de inervación, situación similar a lo descrito en las investigaciones de Linell, Lepage et al. (2005) y Canovas et al. Independiente de lo anterior, nuestros hallazgos difieren de lo descrito por Gunther et al. (1992), ya que en un $75 \%$ de su muestra este músculo presento un solo ramo, situación que nosotros solo encontramos en un $26 \%$. Asímismo, nuestros hallazgos son menores a los descritos por Sunderland \& Ray, ya que a pesar de que también se observó que este músculo recibió 
ramos después de originados los ramos para los otros músculos de este plano superficial, en un $65 \%$ de su muestra este músculo recibió entre 3 y 7 ramos. Asociado con lo anterior y a pesar de Rauber, Poirier \& Charpy y Rouvière $\&$ Delmas, coinciden con nuestra descripción de ramos para éste músculo en la región cubital; Llusá et al. señalan que este músculo sólo recibe ramos desde nervio mediano, una vez que éste ya transitó por la arcada formada por las cabezas de origen de dicho músculo. A lo anterior, Williams et al. agrega que cuando se presentan ramos adicionales a los que surgen en la región cubital, éstos se vinculan con el vientre muscular que tiene este músculo para el dedo índice, situación que coincide con lo observado en el $100 \%$ de los ramos R2 y R3 en nuestro estudio. Dicha condición descrita para el dedo índice podría estar asociada a una mayor independencia funcional presente este dedo, lo que sumado al número de músculos extensores, permite desarrollar una condición muscular solo comparable con el dedo pulgar.

Respecto del origen de los ramos para el músculo flexor superficial de los dedos, el estudio de Sunderland \& Ray, indica que el $70 \%$ de los casos, estos ramos se originaron del nervio mediano, y el $30 \%$ restante, lo hace desde el nervio interóseo anterior. Dicho dato difiere sustancialmente con la gran mayoría de nuestros casos, en la cual el R1 surgió desde el nervio mediano; situación semejante a las planteadas por Liu et al., en la cual tan solo el $10 \%$ de estos ramos surgieron desde el nervio interóseo anterior.

Sunderland \& Ray, también aportaron datos comparables respecto al formato de origen de los ramos para este músculo, en donde en el $75 \%$ de los casos sus ramos surgieron desde un tronco común, siendo lo más frecuente (35 $\%$ ), un origen común con el músculo flexor radial del carpo; en cambio, en nuestro estudio el $87 \%$ de los casos el ramo R1 y la totalidad de los ramos R2 y R3 se originaron de forma independiente. Así mismo, establecieron que en el 95 $\%$ de los casos, estos ramos surgieron distal a la LBE, cifra que concuerda con nuestro $98 \%$ de casos.

En lo que respecta a la distancia del punto de origen del primer ramo para el músculo flexor superficial de los dedos, Gunther et al., señalaron que el promedio alcanzó $7,0 \mathrm{~cm}$ distal a la LBE, cifra que para Linell se establece en los $12 \mathrm{~cm}$ distal a la LBE, ambos promedios, superan al obtenido en nuestra investigación (5,73 cm distal a la LBE), pero supera a los $3,5 \mathrm{~cm}$ distal a la LBE. que Canovas et al., exponen en su investigación.

Respecto del punto de origen de los ramos analizados, lo descrito por Liu et al. coincide en lo que respecta a la ubicación del origen de estos ramos, resultando que en el 90 $\%$ de nuestra muestra, R1 se originó en el tercio proximal, en $60 \%$ de R2 se originaron en el tercio medio y el $100 \%$ de los ramos R3 que surgieron entre la mitad distal del tercio medio y la mitad proximal del tercio distal.

En relación a la distribución de los puntos motores, Linell señaló que la ubicación de los puntos motores se extiende en un intervalo que se dispone entre los 10 hasta los $23 \mathrm{~cm}$, con una distancia promedio de $13 \mathrm{~cm}$ distal a la LBE; dicha cifra supera nuestro promedio de $10,44 \mathrm{~cm}$, con un intervalo que va desde los 7,57 y hasta los $16,87 \mathrm{~cm}$, distal a la misma línea. Sin embargo, estas mediciones son cercanas a las expuestas en el estudio de Lepage et al., quien señaló que los puntos de penetración de este músculo se presentaron en un intervalo que se presentó entre los 6,25 y los 14,5 $\mathrm{cm}$, distal a la línea de referencia.

En lo que respecta a la ubicación de estos puntos motores según los tercios del antebrazo, en el caso de los estudios de Llusá et al. y Liu et al., este músculo presenta una amplia distribución que abarcan el tercio medio e incluso el tercio distal del antebrazo. Al respecto, nuestra investigación no coincide plenamente con lo descrito por estos autores, ya que para el caso de los ramos R1, el $66 \%$ de los casos, este ramo penetró al músculo flexor superficial de los dedos en el tercio proximal del antebrazo, de los cuales, el $85 \%$ lo hizo en la mitad distal de dicho tercio. En cambio, donde sí se establecen coincidencias es en los casos de los ramos R2, que fue el que presentó la mayor variabilidad, ubicando su punto motor $63 \%$ de los casos en el tercio medio, de los cuales, el $57 \%$ lo hizo en mitad distal del citado tercio. La misma disposición presentó el ramo R3, de los cuales en el $60 \%$, ubicó su punto de penetración en la mitad distal del tercio medio, dejando al $40 \%$ restante, como aquellos ramos que establecieron dicho punto motor en la mitad proximal del tercio distal del antebrazo.

En relación a la determinación de los patrones de distribución, un $71 \%$ de la muestra presentó una secuencia que comenzó con la presencia de ramos para el músculo pronador redondo, para distalmente proporcionar ramos a los músculos palmar largo y flexor radial del carpo. A esta secuencia se sumó la presencia del nervio interóseo anterior, el cual se originó proximal a los ramos que inervaron al músculo flexor superficial de los dedos. Dicha descripción no coincide con lo planteado por autores como Cruveilhier, Rouvière \& Delmas y Testut \& Latarjet, quienes señalaron la presencia de un ramo para la capa muscular superficial del compartimiento anterior del antebrazo, el que se divide en varios ramos, que inervan los músculos pronador redondo, flexor radial del carpo, palmar largo y flexor superficial de los dedos. Así mismo, en el $87 \%$ de los casos de nuestra muestra, el músculo flexor superficial de los dedos fue el cuarto músculo de este plano superficial en recibir ramos, 
dato que coincide con lo expuesto por Gunther et al., quienes plantearon separar los ramos que se originan del nervio mediano en 4 grupos, de los cuales el primero estuvo formado por ramos para el músculo pronador redondo, el segundo, incluía ramos destinados a los músculos palmar largo, flexor radial del carpo y flexor superficial de los dedos. El tercer grupo estuvo formado por el nervio interóseo anterior para finalmente indicar que el cuarto grupo estuvo formado por ramos adicionales destinados al músculo flexor superficial de los dedos, disposición descrita de forma similar en nuestro patrón I (85\%).

El estudio de Chantelot et al. (1999), presenta concordancias con nuestro patrón II (15\%), el cual incluyó dos ramos para el músculo pronador redondo y tronco común para los músculos flexor radial del carpo y palmar largo, dejando en un tercer lugar de la secuencia al ramo destinado al músculo flexor superficial de los dedos. Este mismo patrón considera la posibilidad de encontrar ramos adicionales para el músculo flexor superficial de los dedos, situación que coincide con los señalado por Rauber, Poirier \& Charpy y Rouvière \& Delmas.

Todo este análisis representa una evidencia importante al momento de considerar cirugías dentro de la cual se intervengan a estos ramos, lo que es coincidente con lo planteado por Fuss \& Wurzl y Liu et al., quienes señalaron que los patrones descritos por textos clásicos resultan insuficientes para la práctica quirúrgica, por lo que el éxito de los tratamientos invasivos está supeditado al conocimiento preciso y detallado de la distribución, número, punto de origen y punto motores de estos ramos.

Esta descripción de variabilidad en la inervación de este músculo también ejerce influencia en la práctica clínica, ya que, ante la presencia de fenómeno de atrapamientos nerviosos, el conocimiento detallado de la distribución nerviosa, determinará el éxito de un proceso de diagnóstico clínico y posterior tratamiento (Olave et al., 2002; Riveros et al., 2016). Por ello y a pesar de que la mayoría de los textos de anatomía describen bien los trayectos nerviosos, no detallan la distribución de sus ramos, ni menos de los lugares de ingreso en el vientre muscular, conocimiento que de ser detallado podría ofrecer beneficios, tanto en procesos invasivos como también en tratamientos de electroestimulación muscular.

\section{AGRADECIMIENTOS}

Agradecemos al ilustrador Alfredo Torres Parra por su contribución gráfica a esta comunicación científica.
RIVEROS, A.; SOUSA-RODRIGUES. C.F. \& OLAVE, E. Innervation of the superficial flexor digitorum muscle. Inervación del músculo flexor superficial de los dedos. Int. J. Morphol., 36(2):768-777, 2018.

SUMMARY: Innervation of the flexor digitorum superficialis muscle (FDS) is controversial. Several authors describe that the branches of this muscle arise independently or from a common trunk that comes from the median nerve, within the ulnar region. Other descriptions indicate the presence of additional branches in the middle and distal third of the forearm. These differences are also reflected when describing the motor points. The aim was to determine the number, location and distribution patterns of the branches for the FDS. 30 forearms of Brazilian adults were used. About the number of branches, $26 \%$ presented a single branch (R1). $57 \%$ presented two branches (R1 and R2) and $17 \%$ presented 3 branches (R1, R2 and R3). Regarding the format of origin, $87 \%$ of the R1 emerged independently, while $13 \%$ emerged from a common trunk. In the case of R2 and R3, $100 \%$ emerged independently. About the points of origin, $90 \%$ of the R1 were originated in the proximal third of the forearm, while $60 \%$ of the R2 emerged from the middle third, and of the $5 \mathrm{R} 3,60 \%$ were originated in the middle third and the remaining two emerged in the distal third. This same variability was present in the motor points distribution. Regarding the distribution patterns of these motor branches, two types were identified. Pattern I was present in $85 \%$ and was characterized by all branches arising at a point distal to the origin of the anterior interosseous nerve (AIN), however, in the type II pattern (15\% of the sample), the first branch was originated proximal to the origin of the AIN.

KEY WORDS: Anatomy; Median nerve; Motor points; Flexor digitorum superficialis muscle.

\section{REFERENCIAS BIBLIOGRAFICAS}

Canovas, F.; Mouilleron, P. \& Bonnel, F. Biometry of the muscular branches of the median nerve to the forearm. Clin. Anat., 11(4):23945, 1998.

Chantelot, C.; Feugas, C.; Guillem, P.; Chapnikoff, D.; Rémy, F. \& Fontaine, C. Innervation of the medial epicondylar muscles: an anatomic study in 50 cases. Surg. Radiol. Anat., 21(3):165-8, 1999.

Cruveilhier, J. Traíte D`Anatomie Descriptive. 4 ed. Paris, Asselin, 1851.

Fuss, F. K. \& Wurzl, G. H. Median nerve entrapment. Pronator teres syndrome. Surgical anatomy and correlation with symptom patterns. Surg. Radiol. Anat., 12(4):267-71, 1990.

Gunther, S. F.; DiPasquale, D. \& Martin, R. The internal anatomy of the median nerve in the region of the elbow. J. Hand Surg., 17(4):648-56, 1992.

Lepage, D.; Parratte, B.; Tatu, L.; Vuiller, F. \& Monnier, G. Extra- and intramuscular nerve supply of the muscles of the anterior antebrachial compartment: applications for selective neurotomy and for botulinum toxin injection. Surg. Radiol. Anat., 27(5):420-30, 2005.

Linell, E. A. The distribution of nerves in the upper limb, with reference to variabilities and their clinical significance. J. Anat., 55(Pt. 23):79-112, 1921.

Liu, J.; Pho, R. W.; Pereira, B. P.; Lau, H. K. \& Kumar, V. P. Distribution 
of primary motor nerve branches and terminal nerve entry points to the forearm muscles. Anat. Rec., 248(3):456-63, 1997.

Llusá, M.; Palazzi, S. \& Valer, A. Anatomía Quirúrgica del Plexo Braquial y de los Nervios Periféricos de la Extremidad Superior. Buenos Aires, Médica Panamericana, 2013.

Olave, E.; Gabrielli, C.; Braga, M. T. T. \& del Sol, M. Biometric aspects of the motor branches of the musculocutaneous nerve to the brachial muscle. Rev. Chil. Anat., 20(2):231-6, 2002.

Poirier, P. \& Charpy, A. Traíte D`Anatomie humaine. Vol. 3. 12 $2^{\mathrm{a}}$ ed. Paris, Masson et Cie, 1904.

Rauber, A. Lehrbuch der Anatomie des Menschen. Vol. 2. $5^{\text {a }}$ ed. Leipzig, Verlag von Arthur Georgi, 1898.

Riveros, A.; Sousa-Rodrigues, C. F. \& Olave, E. Origin and distribution of the motor branches for the superficial muscles of the anterior region of the forearm in brazilian individuals. Int. J. Morphol., 34(1):356-64, 2016.

Rouvière, H. \& Delmas, A. Anatomía Humana: Descriptiva, Topográfica y Funcional. Vol. 3. 11 ${ }^{\mathrm{a}}$ ed. Barcelona, Masson, 2005.

Sappey, P. C. Traitè D`Anatomie Descriptive. Vol. 3. 4a ed. Paris, Lecrosnier et Babé, 1889.

Sunderland, S. \& Ray, L. J. Metrical and non-metrical features of the muscular branches of the median nerve. J. Comp. Neurol., 85(2):191203, 1946.

Testut, L. \& Latarjet, A. Tratado de Anatomía Humana. Vol. 3. $9^{\mathrm{a}}$ ed. Barcelona, Salvat, 1972.

Williams, P. L.; Bannister, L. H.; Berry, M. M.; Collins, P.; Dyson, M.; Dussek, J. E. \& Ferguson, M. W. J. Anatomía de Gray. Bases Anatómicas de la Medicina y la Cirugía. Vol 2. 38 a ed. Madrid, Churchill Livingstone-Harcourt Brace, 1998.
Dirección para correspondencia:

Klgo. Mg. Andrés Riveros Valdes

Depto. de Ciencias Morfológicas

Universidad San Sebastián

Sede Concepción

Lientur 1457

C.P: 4080871

Concepción

CHILE

Email: andres.riveros@uss.cl

Recibido : 19-12-2017

Aceptado: 25-03-2018 